Journal of Quantitative Spectroscopy \&

Radiative Transfer 99 (2006) 165-174
Journal of Quantitative Spectroscopy \& Radiative Transfer

www.elsevier.com/locate/jqsrt

\title{
Observation of multiply ionized plasmas with dominant bound electron contribution to the index of refraction
}

\author{
J. Filevich ${ }^{\mathrm{a}, *}$, J.J. Rocca ${ }^{\mathrm{a}}$, M.C. Marconi ${ }^{\mathrm{a}}$, S.J. Moon ${ }^{\mathrm{b}}$, J. Nilsen ${ }^{\mathrm{b}}$, J.H. Scofield ${ }^{\mathrm{b}}$, \\ J. Dunn ${ }^{\mathrm{b}}$, R.F. Smith ${ }^{\mathrm{b}}$, R. Keenan ${ }^{\mathrm{b}}$, J.R. Hunter ${ }^{\mathrm{b}}$, V.N. Shlyaptsev ${ }^{\mathrm{c}}$ \\ ${ }^{\mathrm{a}}$ NSF ERC for Extreme Ultraviolet Science and Technology and Department of Electrical and Computer Engineering, \\ Colorado State University, Fort Collins, CO 80523, USA \\ ${ }^{\mathrm{b}}$ Lawrence Livermore National Laboratory, Livermore, CA 94550, USA \\ ${ }^{\mathrm{c}}$ Department of Applied Science, University of California Davis-Livermore, Livermore, CA 94451, USA
}

Accepted 26 April 2005

\begin{abstract}
We report anomalous fringe shifts observed in soft X-ray laser interferograms of laser-created $\mathrm{Al}$ plasmas. This clear experimental evidence shows that the contribution of bound electrons can dominate the index of refraction of laser-created plasmas at soft X-ray wavelengths, resulting in values greater than 1. The comparison of measured and simulated interferograms shows that this results from the dominant contribution of low-charge ions to the index of refraction. This usually neglected bound electron contribution can affect the propagation of soft X-ray radiation in plasmas and the interferometric diagnostics of plasmas for many elements.
\end{abstract}

(C) 2005 Elsevier Ltd. All rights reserved.

Keywords: Interferometry; Soft X-ray laser; Index of refraction; Plasmas; Anomalous dispersion

\section{Introduction}

In multiply ionized laser-created plasmas the index of refraction is usually calculated assuming that the contribution of bound electrons is negligible compared with that of free electrons. For

\footnotetext{
${ }^{*}$ Corresponding author.

E-mail address: rage@engr.colostate.edu (J. Filevich).
} 
example, in all plasma interferometry experiments to date that used soft-X-ray laser probes, the spatial distribution of the electron density has been obtained assuming that the index of refraction is determined by the density of free electrons [1-7]. In partially ionized plasmas, two color interferometry is often used to separate the contribution to the index of refraction of neutral atoms and free electrons [8].

In this paper we present soft X-ray laser interferometry data obtained with a picosecond $14.7 \mathrm{~nm}(84.4 \mathrm{eV})$ laser probe that shows clear evidence that bound electrons can dominate the index of refraction in the late stages of the evolution of an Al plasma created by a high-power laser. The interferograms show that the fringes, late in the plasma evolution, in the periphery of the plasma and close to the target surface bend toward the target. In the experiment, the direction of these fringe shifts is indicative of an index of refraction greater than 1 (hereafter referred as negative fringe shifts). In contrast, at earlier times all the fringes are observed to bend away from the target (positive fringe shifts). The observation of similar negative fringe shifts was previously reported from an independently realized Al laser-created plasma soft X-ray laser interferometry experiment at $13.9 \mathrm{~nm}$ [9]. Analysis of our data, with the assistance of hydrodynamic simulations, indicates that late in the plasma evolution the contribution of bound electrons dominates the index of refraction, causing the observed negative fringe shifts in the periphery regions of the plasma. Herein we expand on results previously published in Ref. [10]. The significance of the result goes beyond the particular case of aluminum, as this effect can strongly affect the index of refraction of many ionized materials at soft X-ray laser wavelengths and needs to be carefully considered when analyzing experiments.

\section{Experimental setup}

The experiment was performed using a transient $14.7 \mathrm{~nm}$ Ni-like Pd soft X-ray laser [11] combined with an amplitude division diffraction grating interferometer (DGI) [4,7]. The soft X-ray laser was pumped by two beams from a chirped pulse amplification laser, the Compact Multipulse Terawatt (COMET) system at LLNL that operates at $1054 \mathrm{~nm}$. An X-ray laser output of a few 10's of $\mu \mathrm{J}$ was achieved by optically pumping a polished Pd target with a sequence of a 600 -ps long pulse $\left(2 \mathrm{~J}, 2 \times 10^{11} \mathrm{~W} \mathrm{~cm}^{-2}\right)$ and a 5 -J energy short pulse of $6.7 \mathrm{ps}$ or $13 \mathrm{ps}$ (FWHM) duration at an incident intensity of $6 \times 10^{13}$ and $3 \times 10^{13} \mathrm{~W} \mathrm{~cm}^{-2}$, respectively. Traveling wave line focus excitation was achieved using a reflection echelon that consists of seven flat mirror segments placed before the focusing optics. Each mirror segment was offset by $0.12 \mathrm{~cm}$ to introduce the traveling wave toward the output of the laser with a delay of $7.7 \mathrm{ps}$ per step. This results in a phase velocity of $c$ along the line focus length and ensures that peak gain conditions are experienced by the propagating X-ray laser photons. The horizontal angular divergence of the soft X-ray laser was measured to be $2.8 \mathrm{mrad}$. The near field and far field beam characteristics were studied and the laser parameters were optimized to obtain beam properties suitable to perform interferometry. The temporal properties of the X-ray laser have been recently measured with a fast X-ray streak camera under the same laser pumping conditions as the interferometry experiments [12]. For saturated X-ray laser output the X-ray duration is typically in the range of 4.5-5.2 ps. With the longer 13 ps pumping pulse, the measured X-ray duration is slightly longer at 5.9 ps [12]. Therefore, the interferograms obtained with this setup have picosecond resolution. 
This short pulse duration permits the acquisition of "snap-shots" of the rapidly evolving plasma, overcoming the blurring of interference fringes that occurs when the electron density profile changes significantly during the duration of the probe pulse. The combination of the robust grating interferometer with the relatively high repetition rate picosecond soft X-ray laser (1 shot every $4 \mathrm{~min}$ ) permitted the acquisition of sequences of interferograms that map the evolution of the electron density distribution in high-density laser created plasmas.

The DGI is set in a skewed Mach-Zehnder configuration as shown in Fig. 1. The light incident on the first grating (G1) is diffracted with approximately equal intensity in the zero and first orders. These two beams that form the two arms of the interferometer are reflected at $2.6^{\circ}$ grazing incidence angle toward a second $7.6 \times 8.5 \mathrm{~cm}^{2}$ diffraction grating (G2) using two $35 \mathrm{~cm}$ long Aucoated mirrors (L1 and L2). Grating G2 recombines the two beams such that they exit the interferometer propagating with a small angular difference, selected to produce fringes of the spacing required by the particular experiment. The gratings have a line spacing of 900 lines $/ \mathrm{mm}$ with a blaze angle of $2^{\circ}$. This grating diffracts the beam into a zero and a first order component of approximately the same intensity when operating at an incidence angle of $6^{\circ}$. For the selected ruling and angle of incidence, the resulting angle between the zero and first diffracted orders is $5.2^{\circ}$. This results in a distance between the two arms of the interferometer at the target position indicated in Fig. 1 to be $\sim 1 \mathrm{~cm}$. There, the phase object of interest, in our case a laser created plasma, is placed. The reflectivity of the gold-coated diffraction gratings is maximized by the small grazing incidence angle used, resulting in an efficiency of $25 \%$ per order on each grating, giving a total throughput for the instrument, excluding the imaging optics, of $\sim 12 \%$. More details on the DGI, including a discussion on the alignment procedure, are given in Ref. [7].

The soft X-ray laser is imaged at the target position onto a $1.33 \times 1.33 \mathrm{~cm}^{2}$ charged-coupled device $(C C D)$ with $1024 \times 1024$ pixels of $13 \times 13 \mu \mathrm{m}^{2}$ size [13]. The imaging optics consist of a $25-\mathrm{cm}$ focal length $\mathrm{Mo} / \mathrm{Si}$ multilayer spherical mirror (S1), set at $5^{\circ}$ off normal incidence, followed by an elongated Au-coated mirror (L3), positioned at a grazing angle of $9^{\circ}$ that relays the beam via a flat $45^{\circ} \mathrm{Mo} / \mathrm{Si}$ multilayer mirror to the $\mathrm{CCD}$ detector located approximately $5.5 \mathrm{~m}$ away

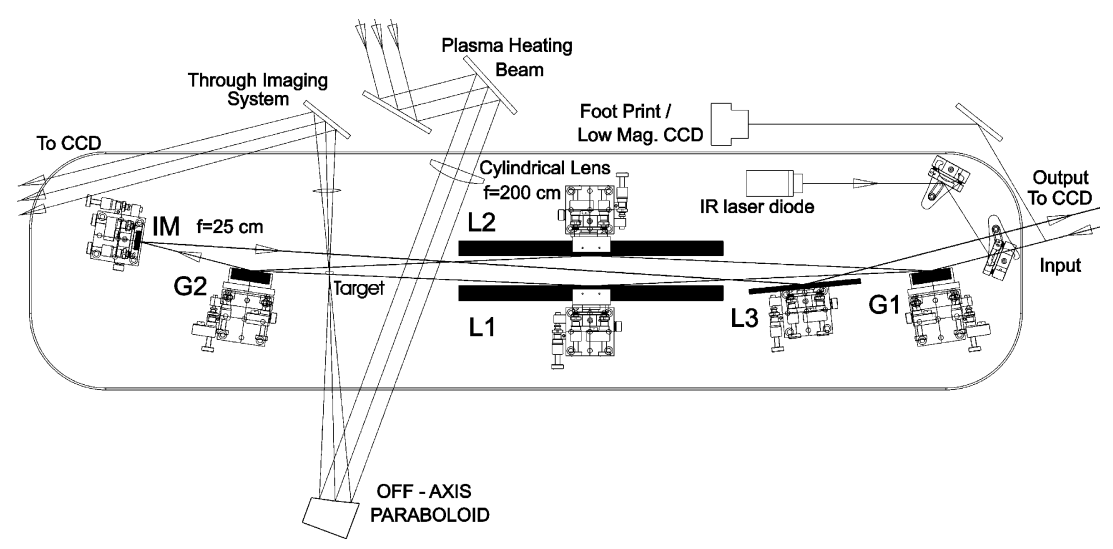

Fig. 1. Experimental setup, depicting the diffraction grating interferometer. The soft X-ray laser enters and exits the interferometer through the lower right side. 
from the imaging mirror. This imaging set up has a total magnification of $22 \times$. The overall system spatial resolution was determined to be $\sim 2 \mu \mathrm{m}$.

\section{Results}

Fig. 2 shows interferograms of an expanding Al laser-produced plasma. The plasmas were heated at an irradiance of $10^{13} \mathrm{~W} \mathrm{~cm}^{-2}$ by focusing a $600-\mathrm{ps}, 3 \mathrm{~J}, 1054 \mathrm{~nm}$ laser into a $3.1 \mathrm{~mm}$ long $\times 12 \mu \mathrm{m}$ wide line focus on a 1-mm long flat Al target. The Al plasma was generated within the path of one arm of the interferometer, and the timing between the heating and probe beams was measured using fast photodiodes. The first four frames corresponding to the earlier time of the plasma evolution ( $0-0.9 \mathrm{~ns})$ show a rapid lateral expansion of the plasma together with the formation of an on-axis density depression. At these times the fringes shift away from the target, even in the central region of the plasma where the on-axis depression is observed. A similar twodimensional feature was observed in soft X-ray laser interferograms of laser-created $\mathrm{Cu}$ plasmas obtained using a 46.9-nm laser [14,15]. In that case the simulations showed that the electron density minimum in the central region of the plasma is the result of pressure equilibrium between the irradiated plasma region and the low temperature side-lobes, created by plasma-radiation
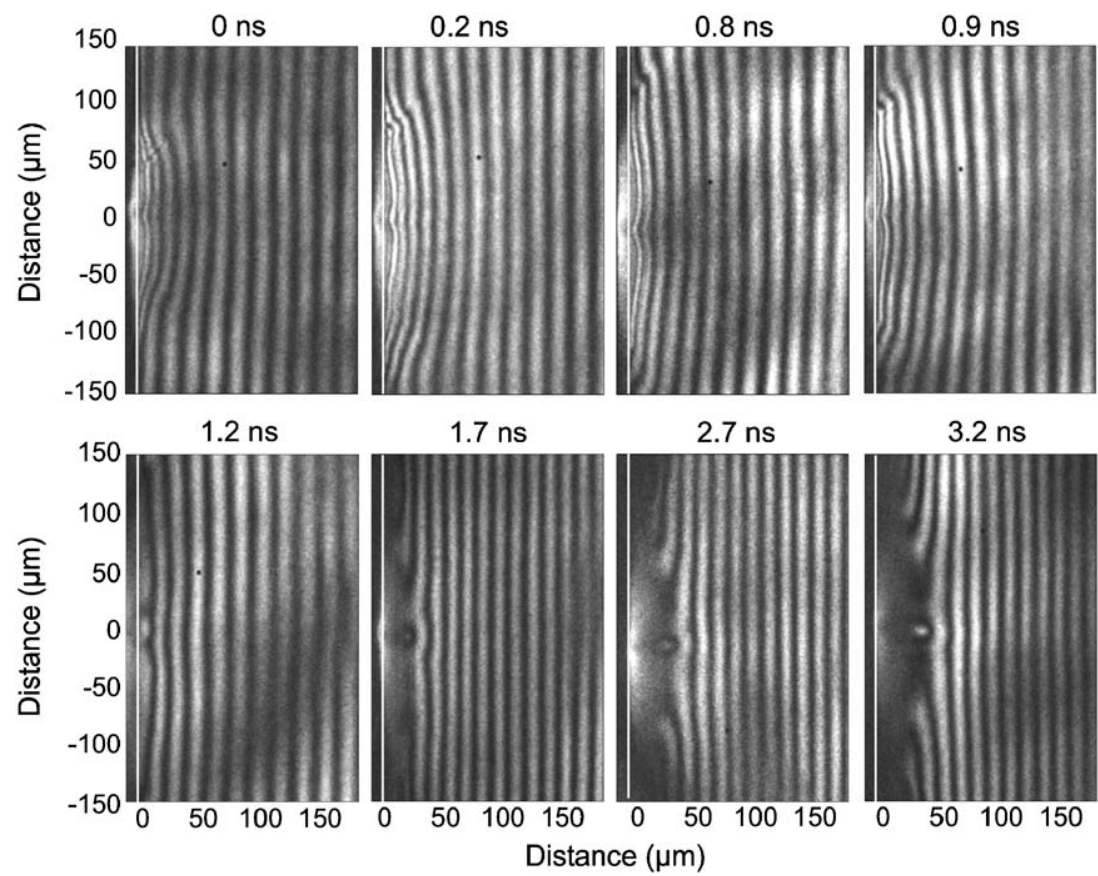

Fig. 2. Sequence of soft X-ray laser interferograms $(\lambda=14.7 \mathrm{~nm})$ of $\mathrm{Al}$ line focus plasmas. The plasmas were generated by a $3-\mathrm{J}$ heating beam focused into a $12 \mu \mathrm{m} \times 3.1 \mathrm{~mm}$ line focus. The targets were $1 \mathrm{~mm}$ long. The times are measured respect to the peak of the $600 \mathrm{ps}$ heating pulse. Fringes that bend toward the target (negative fringe shifts) are observed in the last two frames in the periphery of the plasma and close to the target. 
induced ablation of the surrounding target region. The last four frames in Fig. 2 show interferograms in which the region close to the target presents increased absorption, an indication of a colder plasma. Importantly, the fringes at the periphery of the plasma shift toward the target, a phenomenon that requires a total index of refraction greater than 1 .

In phase shift interferometry the number of fringe shifts, given by $N_{\mathrm{f}}=\frac{1}{\lambda} \int_{0}^{L}(1-\eta) \mathrm{d} l$, is negative when the index of refraction $(\eta)$ of the plasma is greater than 1 . The contribution of the free electrons to the plasma index of refraction is always less than 1 , as determined by $\eta=\left(1-n_{\mathrm{e}} / n_{\text {crit }}\right)^{1 / 2}$, where $n_{\text {crit }}=5 \times 10^{24} \mathrm{~cm}^{-3}$ is the critical density for $\lambda=14.7 \mathrm{~nm}$. This suggests that the contribution of bound electrons to $\eta$ is dominant and greater than one, in the region where negative fringe shifts are observed. The effect was not observed in interferograms of plasmas we generated with similar plasma heating beam conditions using several other target materials (Ti, Cr, Pd, Mo, Au). As an example, Fig. 3 shows interferograms for Al, Ti and Pd plasmas, obtained at approximately the same late time in the plasma evolution as the last frame in Fig. 2, 3 ns after the peak of the heating laser pulse. While the corresponding Al interferogram shows negative fringe shifts on the periphery of the plasma, the Ti and Pd interferograms show only positive fringe shifts.

A possible explanation for this difference can be found reviewing the values of the neutral atom's real component of the scattering factor $f_{1}^{0}$ at $14.7 \mathrm{~nm}$, as shown in Fig. 4 . From all elements with atomic number $(Z)$ less than $54, \mathrm{Al}$ is the only one that has a negative $f_{1}^{0}[16,17]$ due to the close proximity of the $84.4 \mathrm{eV}$ photon energy to the Al L-shell absorption edge. Some elements with $Z \geqslant 54$, including I, Xe, Cs, Ba, Fr, Ra, Ac, also have a negative scattering factor at this wavelength. This negative scattering factor translates into an index of refraction greater than one $\left(\eta=\left(1-\left(r_{\mathrm{e}} \lambda^{2} n_{\mathrm{a}} f_{1}^{0}\right) / \pi\right)^{1 / 2}\right.$ where $n_{\mathrm{a}}$ is the density of neutral atoms, $\lambda$ is the probe beam wavelength and $r_{\mathrm{e}}$ is the classical electron radius). This suggests that significant densities of neutral

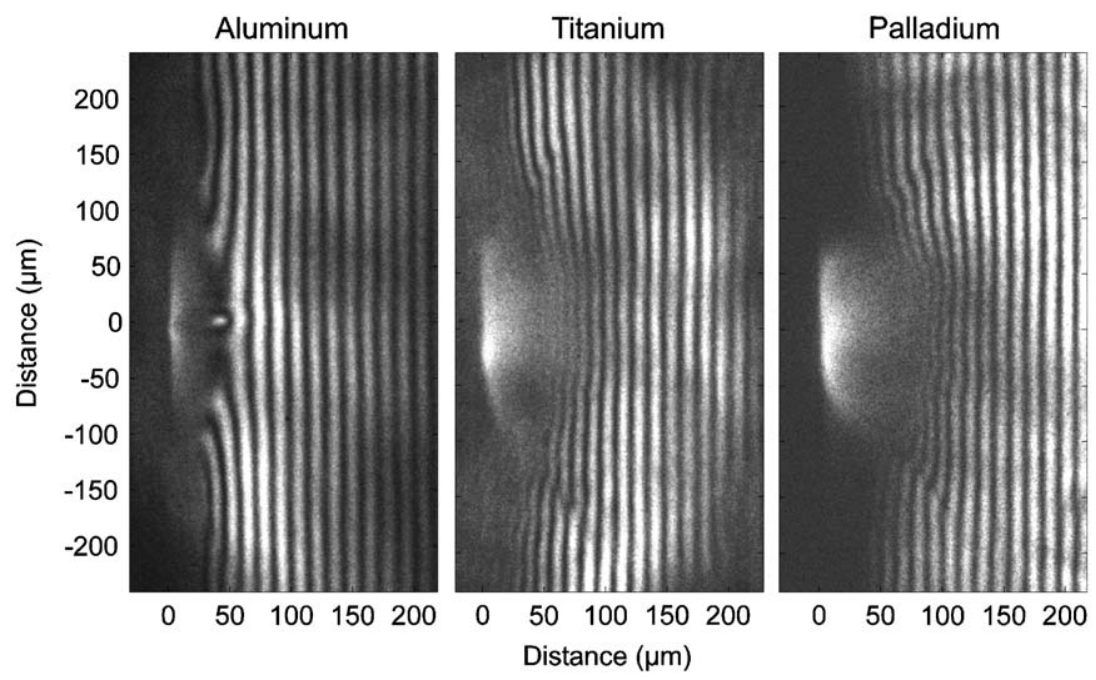

Fig. 3. Comparison of interferograms obtained 3 ns after the peak of the heating laser pulse for $\mathrm{Al}$, Ti and $\mathrm{Pd}$ targets for heating conditions similar as those used to obtain the Al interferograms in Fig. 2. 


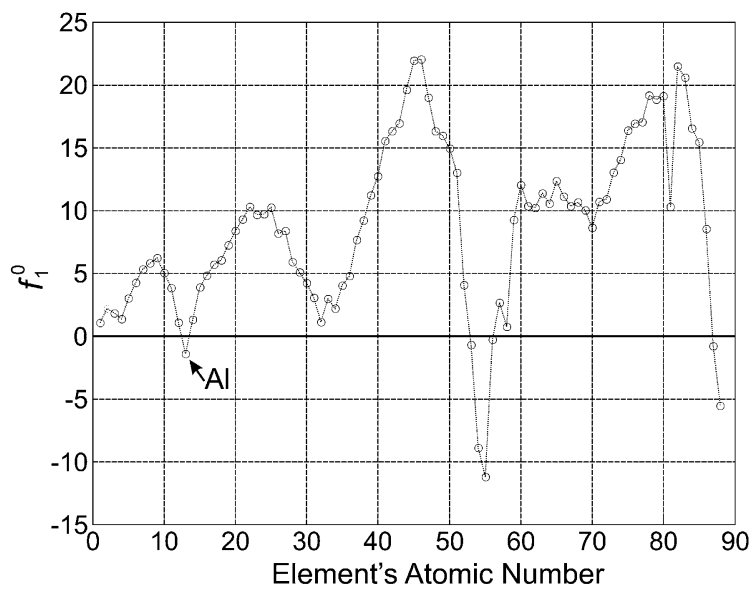

Fig. 4. Scattering factor for neutral atoms $f_{1}^{0}$ vs element's atomic number at $84.4 \mathrm{eV}$ taken from Henke et al. [16] and Gullikson et al. [17].

Table 1

Contribution of the free and bound electrons to the index of refraction

\begin{tabular}{ll}
\hline Ion species & Effective scattering factor $f_{Z}^{\star}$ \\
\hline Al I & -0.85 \\
Al II & -4.19 \\
Al III & -3.54 \\
Al IV & -1.80 \\
Al v & 0.84 \\
Al VI & 3.54 \\
Al VII & 5.30 \\
Al VIII & 6.73 \\
Al IX & 8 \\
Al x & 9.18 \\
Al XI-Al XIV & $\#$ of free electrons \\
\hline
\end{tabular}

Al atoms, for which the L-shell electrons have a significant contribution to $\eta$, will cause negative fringe shifts. However, the high photoionization cross-section of neutral $\mathrm{Al}$ and the fact that the probe beam is not completely absorbed in the region where negative fringe shifts are observed indicate that the neutral density of this plasma should be negligible and that the negative fringe shifts are in fact caused by bound electrons on ions. Therefore, the real component of the index of refraction $f_{1 Z}^{0}$ was computed for the different ions present in the plasma. The calculation uses the best data available at this time with the possibility of further improvements in the future.

We define the "effective" $f_{Z}^{*}$ value as $f_{Z}^{\text {bound }}+f_{Z}^{\text {free }}$ that includes the separate contributions of free and bound electrons, where $f_{Z}^{\text {free }}$ is the number of free electrons corresponding to the ion of charge $Z$ with the forward scattering superscript (0) and real part (1) subscript omitted. We calculated the values for Al I-Al IX and are shown in Table 1. Details on this calculation can be 
found in Ref. [10,18]. When the $f_{Z}^{*}$ values differ from $Z$ (the number of free electrons corresponding to that ion) the contribution of the bound electrons is significant. These $f_{Z}^{*}$ values were used to calculate the total plasma index of refraction $\eta=\left(1-\left(\left(\sum_{Z} n_{Z} f_{Z}^{*}\right) / n_{\text {crit }}\right)^{1 / 2}\right.$, where $n_{Z}$ is the density of ions with charge $Z$ obtained from hydrodynamic simulations which selfconsistently solve hydrodynamics and ionization kinetics equations [19]. All ions with charge up to $Z=5$ were found to make a significant negative contribution to the index of refraction. The computed negative values of $f_{Z}^{*}$ for $\mathrm{Al} \mathrm{I}-\mathrm{Al}$ IV suggest that the negative fringe shifts observed at the late times are due to the presence of a relatively high density of these low charge ions. Moreover, ions up to Al vil make a significant contribution to the index of refraction. It should be noticed that this phenomenon does not require a close resonance and affects a broad range of wavelengths due to numerous bound-bound and bound-free transitions in many ionization stages. This is supported by the observation of negative fringe shifts in Al plasmas at two different probe beam wavelengths: $13.9 \mathrm{~nm}$ [9] and $14.7 \mathrm{~nm}$ (the current experiments).

To confirm this interpretation of the data, the 2-dimensional (2-D) ion and electron density distributions for the plasmas of Fig. 2 was computed with the hydrodynamics simulation. The simulations were performed using flux-limited heat transport, multi-group radiation diffusion, and a detailed hydrogenic configuration atomic model with non-LTE physics for temperatures above $50 \mathrm{eV}$. The simulations were conducted using the measured temporal and spatial profiles of the laser beam used to heat the plasma. Fig. 5 shows 2-D maps of the computed electron density (contours) and the corresponding average ion charge distributions and Fig. 6 shows the computed electron temperature 2-D maps. The electron density distributions measured during the early times of the expansion, including the lateral expansion and on-axis depression, are well reproduced by the simulation. This indicates that the assumption that the free electrons dominate the contribution to the index of refraction is valid at the early times, as is expected from the fact that at those times the plasma is hot and only highly ionized species are present. At later times, corresponding to the last two frames of Fig. 2, the simulations show that the degree of ionization
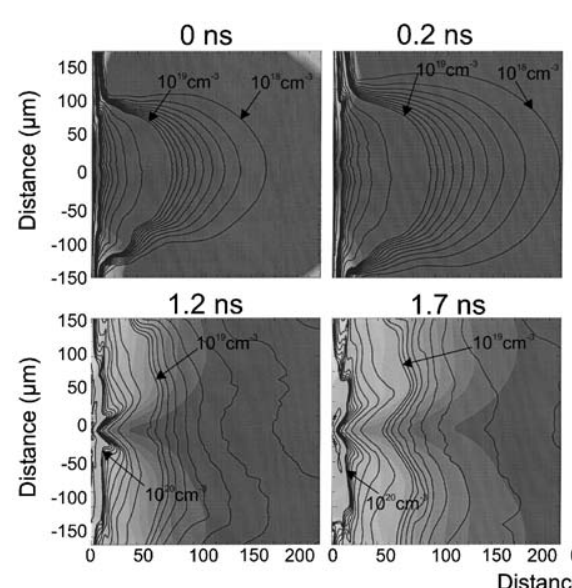

$1.7 \mathrm{~ns}$
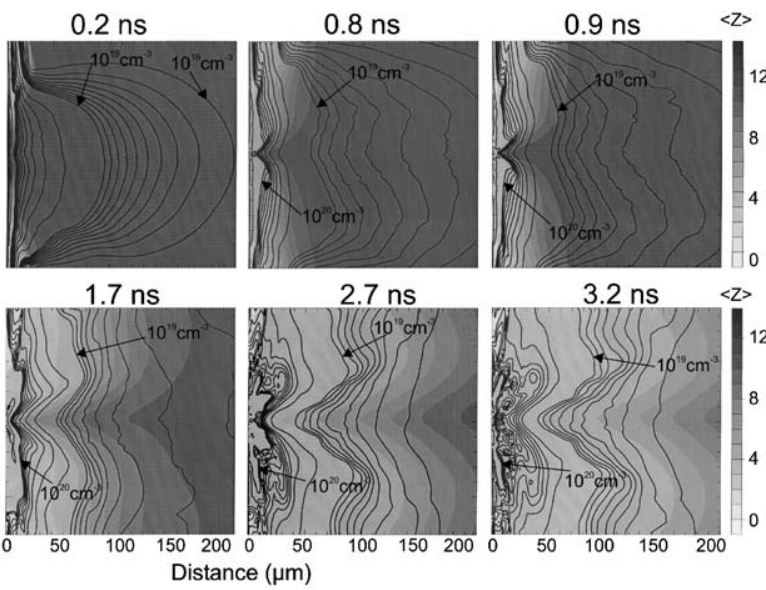

Fig. 5. Sequence of simulated electron density contours shown as lines to indicate the gradients and mean ionization distribution maps computed using the LASNEX code for an Al plasma created with the irradiation conditions of Fig. 2. 
in the plasma periphery decreases as the plasma cools, resulting in significant concentrations of $\mathrm{Al} \mathrm{II-Al} \mathrm{IV} \mathrm{ions.} \mathrm{The} \mathrm{simulation} \mathrm{results} \mathrm{were} \mathrm{used} \mathrm{to} \mathrm{synthesize} \mathrm{the} \mathrm{interferograms} \mathrm{that} \mathrm{are}$ shown in Fig. 7. These synthetic interferograms were calculated taking into account the

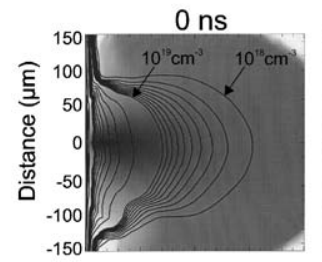

$1.2 \mathrm{~ns}$

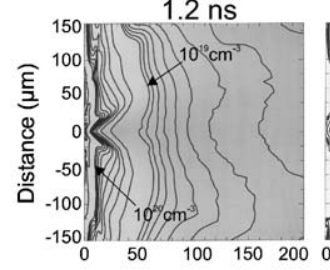

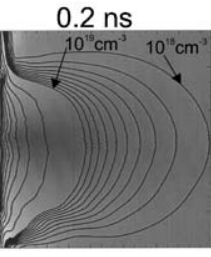

$1.7 \mathrm{~ns}$

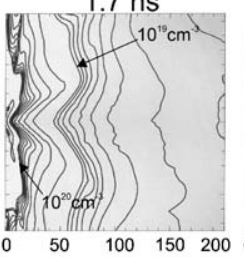

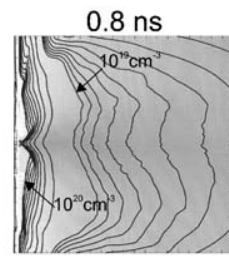

$2.7 \mathrm{~ns}$

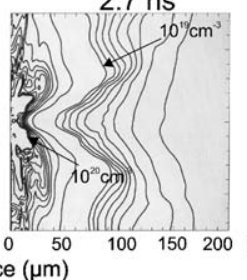

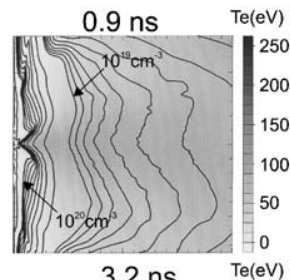

$3.2 \mathrm{~ns} \quad \mathrm{Te}(\mathrm{eV})$

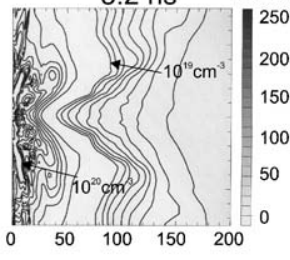

Fig. 6. Sequence of simulated electron density contours shown as lines to indicate the gradients and Electron temperature distribution maps computed using the LASNEX code for an Al plasma created with the irradiation conditions of Fig. 2.
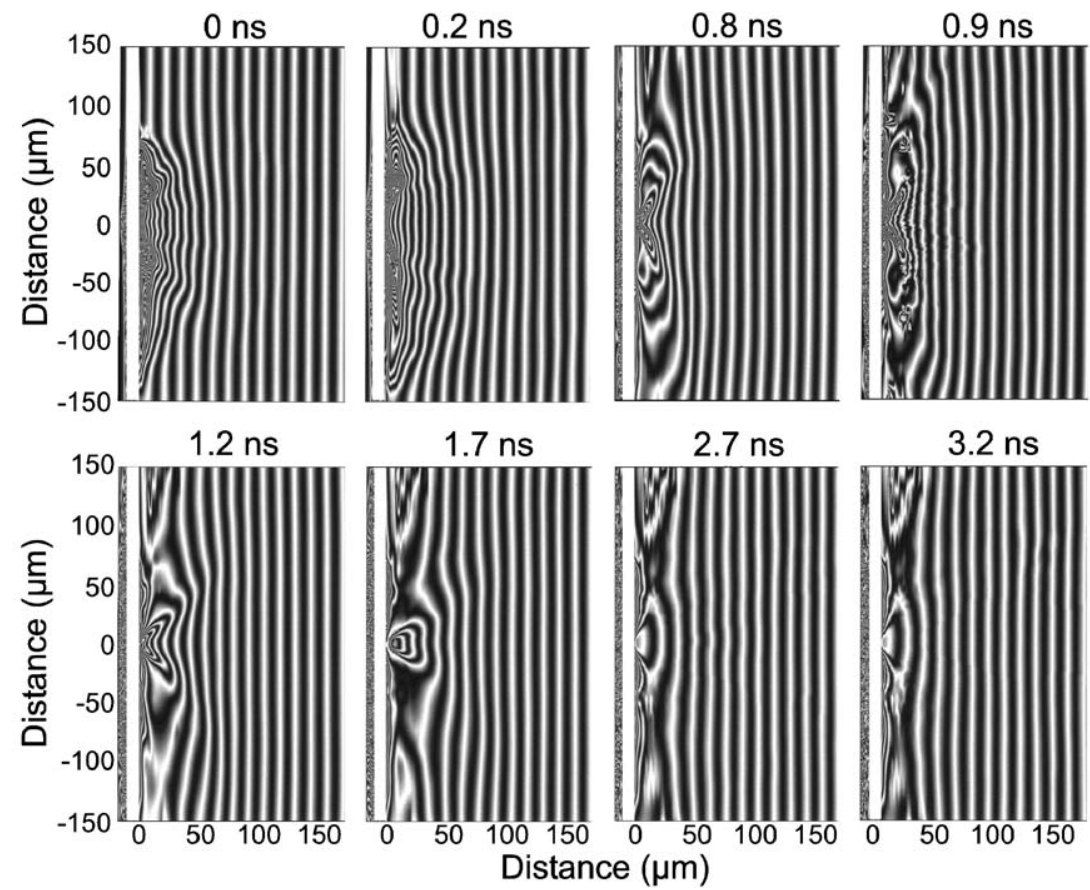

Fig. 7. Synthesized interferograms computed using the calculated electron and ion densities from Fig. 5 and the calculated scattering factors for Al ions. 
contributions to the index of refraction from free electrons and $\mathrm{Al}$ ions using the computed "effective" $f_{Z}^{*}$ factors and the electron density and ion density distributions computed with the simulation. The synthesized interferogram corresponding to $0-0.9 \mathrm{~ns}$ delay show the central density depression and the lateral expansion observed in the measurements, and the late interferograms at $1.2-3 \mathrm{~ns}$ show the observed negative fringe shifts in the periphery of the plasma, in good agreement with the experiment. In the simulations, the negative fringe shifts appear slightly earlier (at $0.8 \mathrm{~ns}$ ) than in the experiment (1.1 ns).

It should be expected that interferograms of the late stages of the evolution of many other laser-created plasmas involving other materials would also be significantly affected by the contribution of bound electrons. Moreover, the importance of the bound electron contribution is not always limited to plasmas with a low mean ion charge. For example, ten times ionized $\mathrm{Pd}$ atoms are computed to have an $f_{1}^{*}$ value that is several times that of the corresponding number of free electrons, with the precise value of $f_{1}^{*}$ depending strongly on the position of the resonance line [18]. It is also important to realize that the relative contribution of bound electron scattering is not always clearly evident in soft X-ray interferograms because at these wavelengths the free and bound electrons often contribute with the same sign to the phase delay. While soft X-ray laser interferometry allows measurements of higher plasma densities because of better spatial resolution, reduced absorption and reduced deflection angles within steep plasma density gradients, neglecting the contribution of bound electrons to the index of refraction could, in some cases, constitute a significant systematic error in the determination of the electron density.

\section{Conclusions}

In conclusion, we have demonstrated that bound electrons can contribute significantly to the index of refraction of multiply ionized plasmas at soft X-ray wavelengths in the vicinity of absorption edges. In the case of an Al plasma the effect causes a dramatic change of sign of the fringe shifts in interferograms acquired using $\lambda=14.7 \mathrm{~nm}$ radiation. The significance of the result goes beyond the particular case of aluminum, as it can significantly affect the index of refraction of many materials at soft X-ray laser wavelengths.

\section{Acknowledgements}

The authors thank Albert Osterheld for continued support. This work was sponsored by the National Nuclear Security Administration under the Stewardship Science Academic Alliances program through DOE Research Grant \# DE-FG03-02NA00062. Part of this work was performed under the auspices of the US Department of Energy by the University of California, Lawrence Livermore National Laboratory through the Institute of Laser Science and Application, under Contract No. W-7405-Eng-48. The CSU researchers also gratefully acknowledge the partial support of the NSF ERC Center for Extreme Ultraviolet Science and Technology, award EEC-0310717. 


\section{References}

[1] Da Silva LB, Barbee Jr TW, Cauble R, Celliers P, Ciarlo D, Libby S, et al. Electron density measurements of high density plasmas using soft X-ray laser interferometry. Phys Rev Lett 1995;74:3991-4.

[2] Rocca JJ, Moreno CH, Marconi MC, Kanizay K. Soft-X-ray laser interferometry of a plasma with a tabletop laser and a Lloyd's mirror. Opt Lett 1999;24:420-2.

[3] Moreno CH, Marconi MC, Kanizay K, Rocca JJ, Uspenskii YA, Vinogradov AV, et al. Soft-X-ray laser interferometry of a pinch discharge using a tabletop laser. Phys Rev E 1999;60:911-7.

[4] Filevich J, Kanizay K, Marconi MC, Chilla JLA, Rocca JJ. Dense plasma diagnostics with an amplitude-division soft-X-ray laser interferometer based on diffraction gratings. Opt Lett 2000;25:356-8.

[5] Smith RF, Dunn J, Nilsen J, Shlyaptsev VN, Moon SH, Filevich J, et al. Picosecond X-ray laser interferometry of dense plasmas. Phys Rev Lett 2002;89:065004.

[6] Rus B, Mocek T, Präg A, Kozlovà M, Hudeček M, Jamelot G, et al. Multi-millijoule, highly coherent X-ray laser at $21 \mathrm{~nm}$ as a routine tool for applications. In: 8th International Conference on X-Ray Lasers; 2002. p. 182-9.

[7] Filevich J, Rocca JJ, Marconi MC, Smith RF, Dunn J, Keenan R, et al. Picosecond resolution soft X-ray laser plasma interferometry. Appl Opt 2004;43:3938-46.

[8] Muraoka K, Maeda M. Laser-aided diagnostics of plasmas and gases. Series in Plasma Physics, IoP Publishing: 2001. p. 154-8.

[9] Tang H, Guilbaud O, Jamelot G, Ros D, Klisnick A, Joyeux D, et al. Diagnostics of laser-induced plasma with soft X-ray $(13.9 \mathrm{~nm})$ bi-mirror interference microscopy. Appl Phys B 2004;78:975-7.

[10] Filevich J, Rocca JJ, Marconi MC, Moon SJ, Nilsen J, Scofield JH, et al. Observation of a multiply ionized plasma with index of refraction greater than one. Phys Rev Lett 2005;94:035005.

[11] Dunn J, Li Y, Osterheld AL, Nilsen J, Hunter JR, Shlyaptsev VN. Gain saturation regime for laser-driven tabletop, transient Ni-like ion X-ray lasers. Phys Rev Lett 2000;84:4834-7.

[12] Dunn J, Smith RF, Shepherd R, Booth R, Nilsen J, Hunter JR, et al. Temporal characterization of a picosecond laser-pumped X-ray laser (for applications). In: Fill EE, Suckewer S, editors. SPIE int soc opt eng proc, vol. 5197. 2003. p. 51-9.

[13] Princeton Instruments PI-SX:1k. Roper Scientific, Inc. 3660 Quakerbridge Road. Trenton, NJ 08619.

[14] Filevich J, Rocca JJ, Jankowska E, Hammarsten EC, Kanizay K, Marconi MC, et al. Two dimensional effects in laser-created plasmas measured with soft-X-ray laser interferometry. Phys Rev E 2003;67:056409.

[15] Rocca JJ, Hammarsten EC, Jankowska E, Filevich J, Marconi MC, Moon SJ, et al. Application of extremely compact capillary discharge soft X-ray lasers to dense plasma diagnostics. Phys Plasmas 2003;10:2031-8.

[16] Henke BL, Gullikson EM, Davis JC. X-ray interactions: photoabsorption, scattering, transmission, and reflection at $E=50-30,000 \mathrm{eV}, Z=1-92$. Atom Data Nucl Data Tables 1993;54:181-342.

[17] Gullikson EM, Denham P, Mrowka S, Underwood JH. Absolute photoabsorption measurements of Mg, Al, and $\mathrm{Si}$ in the soft-X-ray region below the L2,3 edges. Phys Rev B 1994;49:16283-8.

[18] Nilsen J, Scofield JH. Plasmas with index of refraction greater than 1. Opt Lett 2004;29:2677-9.

[19] Zimmerman GD, Kruer WL. Numerical simulation of laser-initiated fusion. Comments Plasma Phys Control Fusion 1975;2:51-61. 